\title{
The impact of sterile neutrinos on CP measurements at long baselines
}

\author{
Raj Gandhi, ${ }^{a, c}$ Boris Kayser, $^{b}$ Mehedi Masud $^{a}$ and Suprabh Prakash ${ }^{a}$ \\ ${ }^{a}$ Harish-Chandra Research Institute, \\ Chhatnag Road, Jhunsi, Allahabad, 211019 India \\ ${ }^{b}$ Theoretical Physics Department, Fermilab, \\ P.O. Box 500, Batavia, IL, 60510 U.S.A. \\ ${ }^{c}$ Neutrino Division, Fermilab, \\ P.O. Box 500, Batavia, IL, 60510 U.S.A.
}

E-mail: raj@hri.res.in, boris@fnal.gov, masud@hri.res.in, suprabhprakash@hri.res.in

Abstract: With the Deep Underground Neutrino Experiment (DUNE) as an example, we show that the presence of even one sterile neutrino of mass $\sim 1 \mathrm{eV}$ can significantly impact the measurements of $\mathrm{CP}$ violation in long baseline experiments. Using a probability level analysis and neutrino-antineutrino asymmetry calculations, we discuss the large magnitude of these effects, and show how they translate into significant event rate deviations at DUNE. Our results demonstrate that measurements which, when interpreted in the context of the standard three family paradigm, indicate $\mathrm{CP}$ conservation at long baselines, may, in fact hide large CP violation if there is a sterile state. Similarly, any data indicating the violation of $\mathrm{CP}$ cannot be properly interpreted within the standard paradigm unless the presence of sterile states of mass $\mathrm{O}(1 \mathrm{eV})$ can be conclusively ruled out. Our work underscores the need for a parallel and linked short baseline oscillation program and a highly capable near detector for DUNE, in order that its highly anticipated results on CP violation in the lepton sector may be correctly interpreted.

KeYwORds: Neutrino Physics, CP violation

ARXiv EPrint: 1508.06275 


\section{Contents}

1 Introduction 1

2 The $3+1$ electron appearance probability in vacuum and matter 2

3 A discussion of neutrino-antineutrino asymmetries in matter 5

4 Event rates at DUNE in the $3+1$ and $3+0$ scenarios 9

$\begin{array}{lll}5 & \text { Implications } & 10\end{array}$

$6 \quad$ Summary and conclusions $\quad 12$

\section{Introduction}

A major goal of present and future long-baseline neutrino oscillation experiments is to establish that leptons violate $\mathrm{CP}$, or else to place a stringent upper limit on any such violation. (For recent status reviews see [1-12].) Our thinking about these experiments usually assumes the standard neutrino paradigm, in which there are just three neutrino mass eigenstates separated by just two independent mass-squared splittings, three mixing angles $\theta_{i j}$, and just one CP-violating phase $\delta_{\mathrm{CP}}$ relevant to oscillation. However, a variety of short-baseline anomalies [13-17] hint at the possible existence of short-wavelength oscillations, driven by one or more $O\left(1 \mathrm{eV}^{2}\right)$ mass-squared splittings that are much larger than the two splittings of the standard paradigm. These short-wavelength oscillations are purportedly already significant when the (Travel distance $L$ )/(Energy $E$ ) of neutrinos in a beam is only $\sim 1 \mathrm{~km} / \mathrm{GeV}$. Of course, they are still present at the far detector of any long-baseline experiment, where $L / E$ is, say, $\sim 500 \mathrm{~km} / \mathrm{GeV}$. In this work, we have explored the consequences of the short-wavelength oscillations - should they be real - for measurements at long baselines, especially measurements that probe CP violation. We find that these consequences could be considerable. For example, it is possible for long-baseline results, interpreted without taking the short-wavelength oscillations into account, to imply that $\mathrm{CP}$ violation is very small or totally absent, when in reality it is very large. In addition, long-baseline measurements interpreted as determining the sole oscillation-relevant CP-violating phase in the standard paradigm could in fact be measuring something else.

The large splittings hinted at by the short-baseline anomalies imply the existence of additional, largely sterile, neutrino mass eigenstates, beyond the three of the standard scenario (referred to as $3+0$ in what follows). These additional mass eigenstates introduce not only additional splittings but also additional mixing angles and phases. For simplicity, we restrict ourselves to the scenario, referred to as $3+1$, with only one additional mass 
eigenstate. In this scenario, there are six mixing angles, and three $\mathrm{CP}$-violating phases that can affect oscillation. Denoting the mass eigenstates of $3+0$, as usual, as $\nu_{1}, \nu_{2}, \nu_{3}$, and the additional mass eigenstate as $\nu_{4}$, and defining the mass-squared splittings as $\delta m_{i j}^{2}=$ $m_{i}^{2}-m_{j}^{2}(\mathrm{i}, \mathrm{j}=1,2,3,4 \& i \neq j)$, we have, according to present data,

$$
\delta m_{41}^{2} \sim \delta m_{42}^{2} \sim \delta m_{43}^{2} \gg\left|\delta m_{31}^{2}\right| \sim\left|\delta m_{32}^{2}\right| \gg \delta m_{21}^{2} .
$$

Since the probability of an oscillation driven by a splitting $\delta m_{i j}^{2}$ is proportional to $\sin ^{2} \Delta_{i j}$, where $\Delta_{i j}=1.27 \times \frac{\delta m_{i j}^{2}\left[\mathrm{eV}^{2}\right] \times L[\mathrm{~km}]}{E[\mathrm{GeV}]}$, when L/E $\sim 500 \mathrm{~km} / \mathrm{GeV}$, the short-wavelength oscillations driven by the large splittings involving $\nu_{4}$ will be averaged to an $\mathrm{L} / \mathrm{E}$ independent value by the finite energy resolution of any realistic detector. But these rapid oscillations are still present and can have a major impact.

We perform our calculations for the $3+1$ scenario as manifested in the proposed Deep Underground Neutrino Experiment (DUNE). ${ }^{1}$ While we do not explore $3+\mathrm{N}$ scenarios with $N>1$, we expect that if the consequences of having one extra neutrino for long-baseline measurements are substantial, those of having more than one must be substantial as well, since the world with one extra neutrino is in a sense a special case of that with more than one.

Previous work examining the effects of sterile neutrinos at long baselines includes several studies of neutrino factories feeding baselines of about $3000 \mathrm{~km}-7500 \mathrm{~km}$, with muon energies in the range $20 \mathrm{GeV}-50 \mathrm{GeV}$, focussing on effects at both near and far detectors [21-25]. More recent work [26] includes a study of effects relevant to T2K [27] and a combined study [28] for T2K, MINOS [29] and reactor experiments. Additionally, issues having some overlap with those addressed here for DUNE have been discussed in [30], and, very recently, in [31].

In section 2 we examine, in the $3+1$ scenario, the probability $P_{\mu e}^{4 \nu}$ of $\nu_{e}$ appearance at the far end of a long-baseline beam of neutrinos born as $\nu_{\mu}$. We derive an analytical expression for $P_{\mu e}^{4 \nu}$ valid in vacuum, and discuss how its qualitative features change when matter effects, which will be large in the case of DUNE, are taken into account. Section 3 focuses on the neutrino-antineutrino asymmetries that are possible at long baselines in the $3+0$ and $3+1$ scenarios, and on how the possible asymmetries in these two scenarios compare. Section 4 presents long-baseline far detector event rates, obtained by performing realistic rate calculations for the two scenarios. Section 5 briefly discusses two important implications of the results presented here. In section 6 , we summarize and discuss what conclusions could possibly be drawn, and what ones could not be drawn, especially concerning $\mathrm{CP}$ violation, from a given set of long-baseline oscillation results, and conclude.

\section{The $3+1$ electron appearance probability in vacuum and matter}

For CPV discovery in long baseline super-beam experiments, the electron neutrino appearance probability $P_{\mu e}$ is crucial. We discuss its analytic form in vacuum for the $3+1$ scenario

\footnotetext{
${ }^{1}$ The inputs we use, and the corresponding references, pertain to the erstwhile Long Baseline Neutrino Experiment (LBNE), which has undergone a new phase of internationalisation and expansion. This has led to a change in the name of the experiment, to DUNE. Nonetheless, it is expected that the configuration we assume here vis a vis fluxes, baseline and energies will remain largely intact [18-20].
} 
prior to discussing the matter case. While it is the latter that is relevant for DUNE in particular, and other long baseline (LBL) experiments at baselines of $O(1000) \mathrm{km}$ in general, the form of the vacuum expression provides a useful template for the identification of terms the importance of which will be accentuated by the presence of matter.

We adopt the following parameterisation ${ }^{2}$ for the PMNS matrix in the presence of a sterile neutrino,

$$
U_{\mathrm{PMNS}}^{3+1}=O\left(\theta_{34}, \delta_{34}\right) O\left(\theta_{24}, \delta_{24}\right) O\left(\theta_{14}\right) O\left(\theta_{23}\right) O\left(\theta_{13}, \delta_{13}\right) O\left(\theta_{12}\right) .
$$

Here, in general, $O\left(\theta_{i j}, \delta_{i j}\right)$ is a rotation matrix in the $i j$ sector with associated phase $\delta_{i j}$. For example,

$$
O\left(\theta_{24}, \delta_{24}\right)=\left(\begin{array}{cccc}
1 & 0 & 0 & 0 \\
0 & \cos \theta_{24} & 0 & e^{-i \delta_{24}} \sin \theta_{24} \\
0 & 0 & 1 & 0 \\
0 & -e^{i \delta_{24}} \sin \theta_{24} & 0 & \cos \theta_{24}
\end{array}\right) ; O\left(\theta_{14}\right)=\left(\begin{array}{cccc}
\cos \theta_{14} & 0 & 0 & \sin \theta_{14} \\
0 & 1 & 0 & 0 \\
0 & 0 & 1 & 0 \\
-\sin \theta_{14} & 0 & 0 & \cos \theta_{14}
\end{array}\right) \text { etc. }
$$

Using the standard formula for a flavour transition oscillation probability, we have, for the $3+1$ case:

$$
\begin{aligned}
P_{\mu e}^{4 \nu}= & 4\left|U_{\mu 4} U_{e 4}\right|^{2} \times 0.5 \\
& -4 \operatorname{Re}\left(U_{\mu 1} U_{e 1}^{*} U_{\mu 2}^{*} U_{e 2}\right) \sin ^{2} \Delta_{21}+2 \operatorname{Im}\left(U_{\mu 1} U_{e 1}^{*} U_{\mu 2}^{*} U_{e 2}\right) \sin 2 \Delta_{21} \\
& -4 \operatorname{Re}\left(U_{\mu 1} U_{e 1}^{*} U_{\mu 3}^{*} U_{e 3}\right) \sin ^{2} \Delta_{31}+2 \operatorname{Im}\left(U_{\mu 1} U_{e 1}^{*} U_{\mu 3}^{*} U_{e 3}\right) \sin 2 \Delta_{31} \\
& -4 \operatorname{Re}\left(U_{\mu 2} U_{e 2}^{*} U_{\mu 3}^{*} U_{e 3}\right) \sin ^{2} \Delta_{32}+2 \operatorname{Im}\left(U_{\mu 2} U_{e 2}^{*} U_{\mu 3}^{*} U_{e 3}\right) \sin 2 \Delta_{32} .
\end{aligned}
$$

In arriving at the above expression, we have only assumed (based on eq. (1.1) above) that $\sin ^{2} \Delta_{4 i}$ averages out to be 0.5 at long baselines, and similarly $\sin 2 \Delta_{4 i}$ averages out to be 0 , when $i=1,2,3$.

After substituting the values of the $U_{\alpha i}$ in terms of the mixing angles, we obtain:

$$
\begin{aligned}
P_{\mu e}^{4 \nu}= & \frac{1}{2} \sin ^{2} 2 \theta_{\mu e}^{4 \nu} \\
& +\left(a^{2} \sin ^{2} 2 \theta_{\mu e}^{3 \nu}-\frac{1}{4} \sin ^{2} 2 \theta_{13} \sin ^{2} 2 \theta_{\mu e}^{4 \nu}\right)\left[\cos ^{2} \theta_{12} \sin ^{2} \Delta_{31}+\sin ^{2} \theta_{12} \sin ^{2} \Delta_{32}\right] \\
& +\cos \left(\delta_{13}\right) b a^{2} \sin 2 \theta_{\mu e}^{3 \nu}\left[\cos 2 \theta_{12} \sin ^{2} \Delta_{21}+\sin ^{2} \Delta_{31}-\sin ^{2} \Delta_{32}\right] \\
& +\cos \left(\delta_{24}\right) b a \sin 2 \theta_{\mu e}^{4 \nu}\left[\cos 2 \theta_{12} \cos ^{2} \theta_{13} \sin ^{2} \Delta_{21}-\sin ^{2} \theta_{13}\left(\sin ^{2} \Delta_{31}-\sin ^{2} \Delta_{32}\right)\right] \\
& +\cos \left(\delta_{13}+\delta_{24}\right) a \sin 2 \theta_{\mu e}^{3 \nu} \sin 2 \theta_{\mu e}^{4 \nu}\left[-\frac{1}{2} \sin ^{2} 2 \theta_{12} \cos ^{2} \theta_{13} \sin ^{2} \Delta_{21}\right. \\
& \left.+\cos 2 \theta_{13}\left(\cos ^{2} \theta_{12} \sin ^{2} \Delta_{31}+\sin ^{2} \theta_{12} \sin ^{2} \Delta_{32}\right)\right]
\end{aligned}
$$

\footnotetext{
${ }^{2}$ This choice, which, at first, appears not to be the most general one which could be made, is motivated by the fact that in any parameterisation, the electron neutrino $3+1$ appearance probability in vacuum turns out to be dependent on only two specific linear combinations of the three independent phases. For simplicity, we have thus incorporated this at the outset using the fact that the first and second row elements $\left(U_{e i}\right.$ and $\left.U_{\mu i}\right)$ in $\mathrm{U}_{\mathrm{PMNS}}^{3+1}$ will not have $\theta_{34}$ or $\delta_{34}$ in them.
} 


$$
\begin{aligned}
& -\frac{1}{2} \sin \left(\delta_{13}\right) b a^{2} \sin 2 \theta_{\mu e}^{3 \nu}\left[\sin 2 \Delta_{21}-\sin 2 \Delta_{31}+\sin 2 \Delta_{32}\right] \\
& +\frac{1}{2} \sin \left(\delta_{24}\right) b a \sin 2 \theta_{\mu e}^{4 \nu}\left[\cos ^{2} \theta_{13} \sin 2 \Delta_{21}+\sin ^{2} \theta_{13}\left(\sin 2 \Delta_{31}-\sin 2 \Delta_{32}\right)\right] \\
& +\frac{1}{2} \sin \left(\delta_{13}+\delta_{24}\right) a \sin 2 \theta_{\mu e}^{3 \nu} \sin 2 \theta_{\mu e}^{4 \nu}\left[\cos ^{2} \theta_{12} \sin 2 \Delta_{31}+\sin ^{2} \theta_{12} \sin 2 \Delta_{32}\right] \\
& +\left(b^{2} a^{2}-\frac{1}{4} a^{2} \sin ^{2} 2 \theta_{12} \sin ^{2} 2 \theta_{\mu e}^{3 \nu}-\frac{1}{4} \cos ^{4} \theta_{13} \sin ^{2} 2 \theta_{12} \sin ^{2} 2 \theta_{\mu e}^{4 \nu}\right) \sin ^{2} \Delta_{21}
\end{aligned}
$$

where,

$$
\begin{aligned}
\sin 2 \theta_{\mu e}^{3 \nu} & =\sin 2 \theta_{13} \sin \theta_{23} \\
b & =\cos \theta_{13} \cos \theta_{23} \sin 2 \theta_{12} \\
\sin 2 \theta_{\mu e}^{4 \nu} & =\sin 2 \theta_{14} \sin \theta_{24} \\
a & =\cos \theta_{14} \cos \theta_{24} .
\end{aligned}
$$

Prior to proceeding, we briefly discuss the allowed ranges for the $3+1$ mixing angles that we have used in our calculations. These have been obtained using the results of [32], which takes all available data on short-baseline (SBL) oscillations and performs a global fit to constrain active-sterile mixing. $\left|U_{e 4}\right|^{2}$ is constrained by $\nu_{e}$ and $\bar{\nu}_{e}$ disappearance searches, and is equal to $\sin ^{2} \theta_{14}$. From [32], the $99 \%$ C.L. limit can be taken to be (with some extrapolation, as the result is given for $95 \%$ ),

$$
\left|U_{e 4}\right|^{2} \in[0,0.1]
$$

which gives

$$
\theta_{14} \in\left[0,20^{\circ}\right]
$$

Similarly, using $\nu_{\mu}, \bar{\nu}_{\mu}$ and neutral current disappearance searches, one can constrain $U_{\mu 4}$ and $U_{\tau 4}$, which are given by,

$$
\left|U_{\mu 4}\right|=\cos \theta_{14} \sin \theta_{24}, \quad\left|U_{\tau 4}\right|=\cos \theta_{14} \cos \theta_{24} \sin \theta_{34} .
$$

The constraints on these elements are found to be,

$$
\left|U_{\mu 4}\right|^{2} \in[0,0.03], \quad\left|U_{\tau 4}\right|^{2} \in[0,0.3] .
$$

These translate to,

$$
\theta_{24} \in\left[0,10^{\circ}\right], \quad \theta_{34} \in\left[0,30^{\circ}\right] .
$$

Apropos eq. (2.3), we note that, as expected, the vacuum appearance probability is independent of the 3-4 mixing angle and the associated CP phase. This important simplification, however, does not carry over to the matter case, as we show below. Secondly, eq. (2.3) contains terms proportional to the sines and cosines of a) the $3+1 \mathrm{CP}$ phase $\delta_{24}$, and b) the sum $\left(\delta_{13}+\delta_{24}\right)$. These are interference terms, involving mixing angles from both the $3+0$ and the $3+1$ sector. In particular, as can be determined by inspection, the terms involving the sine and cosine of the sum of $\delta_{13}$ and $\delta_{24}$ can be significantly large 
and lead to appreciable changes in both the amplitude of the overall probability and the extent of $\mathrm{CP}$ violation. These contributions become all the more significant once matter effects are large. We discuss this in more detail below, both in this section and the next.

The matter eigenstates bring about a dependence on all mixing angles and phases. Specifically, unlike the vacuum case, the 3-4 mixing angle and its associated phase are no longer quiescent, and the $3+1$ electron neutrino appearance probability exhibits a significant dependence on them. This is illustrated by figure 1, where we have used the General Long Baseline Experiment Simulator (GLoBES) $[33,34]$ to generate the plots. The left panel shows the variation of $P_{\mu e}^{4 \nu}$ with energy for no CP violation (all three Dirac CP phases set to zero) and four different values of the 3-4 mixing angle. While the variation due to $\theta_{34}$, shown in the left panel, is not very large, the effect of varying $\delta_{34}$ (while keeping $\theta_{34}$ fixed, right panel) within its allowed range is quite significant. It is also striking that these large effects on the probability in the presence of matter are brought about by parameters which are completely absent in the vacuum expression (eq. (2.3)) and which (in our chosen parametrization) play no role in SBL situations. They illustrate the important role matter plays in invoking and enhancing the effects of sterile states at long baselines. In these plots, all other parameters are fixed as described in the caption.

Figure 2 emphasizes the dependencies discussed above from a slightly more general perspective. In the right $(3+1)$ panel, the significant differences between the solid and dashed lines of a given colour emphasize the role played by matter, while the equally significant differences between the blue and red dashed (solid) lines demonstrate the important role played by $\mathrm{CP}$ violating phases at long baselines in matter (vacuum) if they are nonzero. Turning to the left $(3+0)$ panel, we note the relatively large differences between these curves and their counterparts in the right panel, underlining the significant effects of sterile neutrinos at the operational baseline for DUNE.

In summary, figures 1 and 2 demonstrate that additional $\mathrm{CP}$ phases related to a $\mathrm{eV}^{2}$ sterile sector play an important role at long baselines. Their effects are heightened by the presence of matter. In addition, parameters related to the sterile sector which are dormant at short baselines and in vacuum-like conditions are no longer inert once baselines are long and matter effects are important.

\section{A discussion of neutrino-antineutrino asymmetries in matter}

The consideration of $\mathrm{CP}$ violation in terms of an asymmetry defined at the probability level provides additional insight into the conclusions which can be reliably drawn from data if we do not know whether $3+0$ or $3+1$ is the choice nature has made. Consider the asymmetry defined as,

$$
A_{\nu \bar{\nu}}^{\alpha \beta}=\frac{P\left(\nu_{\alpha} \rightarrow \nu_{\beta}\right)-P\left(\bar{\nu}_{\alpha} \rightarrow \bar{\nu}_{\beta}\right)}{P\left(\nu_{\alpha} \rightarrow \nu_{\beta}\right)+P\left(\bar{\nu}_{\alpha} \rightarrow \bar{\nu}_{\beta}\right)} \equiv \frac{\Delta P_{\alpha \beta}}{P\left(\nu_{\alpha} \rightarrow \nu_{\beta}\right)+P\left(\bar{\nu}_{\alpha} \rightarrow \bar{\nu}_{\beta}\right)} .
$$

We begin by noting an important difference between the $3+0$ and $3+1$ scenarios with respect to the numerator $\Delta P_{\alpha \beta}$ of $A_{\nu \bar{\nu}}^{\alpha \beta}$. In vacuum, CPT invariance implies that $P\left(\nu_{\beta} \rightarrow \nu_{\alpha}\right)=P\left(\bar{\nu}_{\alpha} \rightarrow \bar{\nu}_{\beta}\right)$, which in turn implies that $\Delta P_{\beta \alpha}=-\Delta P_{\alpha \beta}$, and in particular 

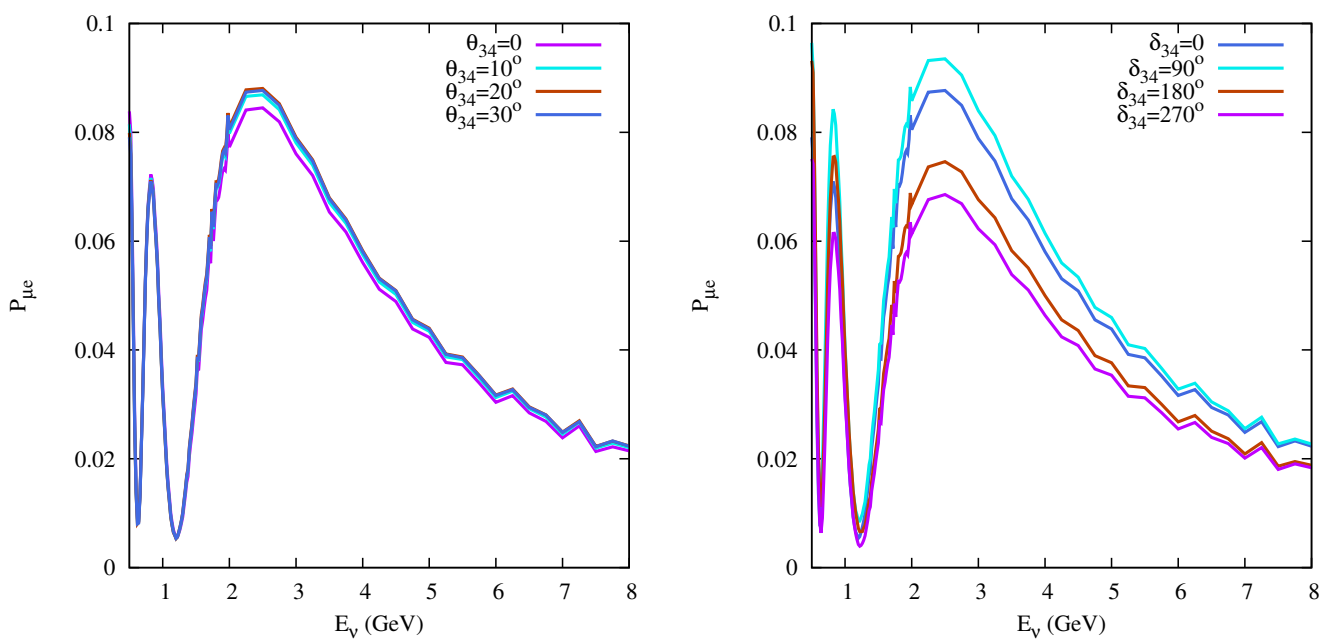

Figure 1. $P_{\mu e}$ vs $E_{\nu}$ in earth matter for $1300 \mathrm{~km}$. Averaging has been done for $\Delta m_{4 i}^{2}$ induced oscillations. In the left panel, the effect of varying $\theta_{34}$ within its allowed range is shown with all the $\mathrm{CP}$ phases kept equal to 0 . In the right panel, we show the effect of varying $\mathrm{CP}$ violating phase $\delta_{34}$ when $\theta_{34}=30^{\circ}$, and the other phases are 0 . For both panels, we set $\theta_{14}=20^{\circ}$ and $\theta_{24}=10^{\circ}$, and the parameters related to the $3+0$ sector at the best-fit values specified in section 4 .

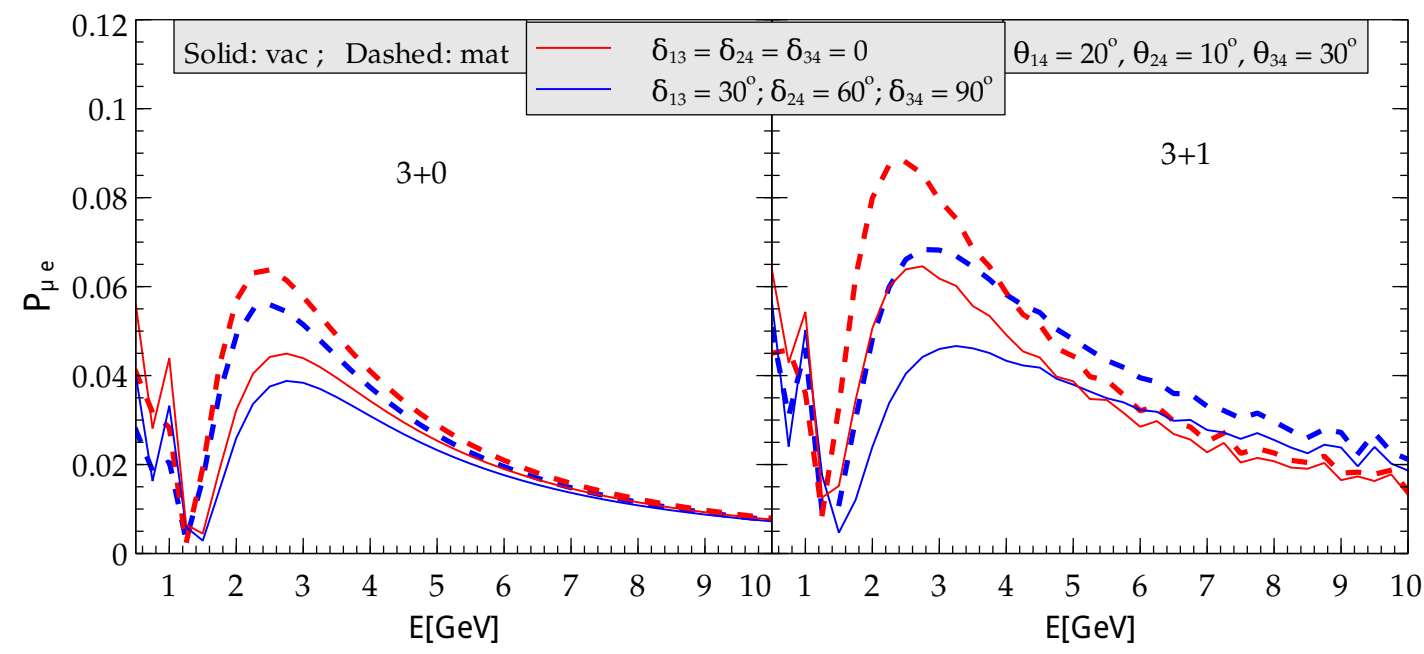

Figure 2. $P_{\mu e}$ (both for vacuum and matter) for $3+0$ (left panel) and $3+1$ (right panel) vs. energy. The red curves represent the $\mathrm{CP}$ conserving case, while the blue ones depict the case with phases set to non-zero fixed values (see the plot label). For the blue curve in the left panel, the sole $3+0$ phase $\delta_{\mathrm{CP}}$ was taken as $30^{\circ}$. Normal hierarchy is taken to be the true hierarchy here, and parameters related to the $3+0$ sector have been set at the best-fit values specified in section 4 .

that $\Delta P_{\alpha \beta}=0$ when $\beta=\alpha$. Thus, when there are only three neutrino flavors, there are only three independent potentially non-zero CP-violating differences $\Delta P_{\alpha \beta}$ to be measured: $\Delta P_{e \mu}, \Delta P_{\mu \tau}$ and $\Delta P_{\tau e}$. Now, conservation of probability implies that for any number of flavors,

$$
\sum_{\beta} P\left(\nu_{\alpha} \rightarrow \nu_{\beta}\right)=1 \quad \text { and } \quad \sum_{\beta} P\left(\bar{\nu}_{\alpha} \rightarrow \bar{\nu}_{\beta}\right)=1
$$


where the sums are over all $\beta$, including $\beta=\alpha$. It follows that $\sum_{\beta} \Delta P_{\alpha \beta}=0$. Then, since $\Delta P_{\alpha \beta}=0$ when $\beta=\alpha$, we conclude that in vacuum,

$$
\sum_{\beta \neq \alpha} \Delta P_{\alpha \beta}=0
$$

When there are only three flavors, this constraint implies that $\Delta P_{e \mu}+\Delta P_{e \tau}=0$ and that $\Delta P_{\mu e}+\Delta P_{\mu \tau}=0$. Since $\Delta P_{\beta \alpha}=-\Delta P_{\alpha \beta}$, it follows that,

$$
\Delta P_{e \mu}=\Delta P_{\mu \tau}=\Delta P_{\tau e}
$$

That is, the three "independent" CP-violating differences are equal. In particular, if there are only three flavors, it is not possible for CP invariance to hold in one oscillation channel, such as $\stackrel{(-)}{\nu_{\mu}} \rightarrow \stackrel{(-)}{\nu_{e}}$, and yet be violated in another channel, such as $\stackrel{(-)}{\nu_{\mu}} \rightarrow \stackrel{(-)}{\nu_{\tau}}$.

This situation changes when there are more than three flavors. For e.g., when there are four flavors, as in the $3+1$ scenario, there are six independent potentially non-zero differences $\Delta P_{\alpha \beta}: \Delta P_{e \mu}, \Delta P_{\mu \tau}, \Delta P_{\tau e}, \Delta P_{e s}, \Delta P_{\mu s}$ and $\Delta P_{\tau s}$, where $s$ refers to the sterile flavor. Now the constraint of eq. (3.2) gives rise only to relations like

$$
\Delta P_{e \mu}=\Delta P_{\mu \tau}+\Delta P_{\mu s}
$$

It is now perfectly possible for $\Delta P_{\mu e}\left(=-\Delta P_{e \mu}\right)$, the CP-violating difference that will be the first to be probed experimentally, to be zero, while the differences $\Delta P_{\mu \tau}$ and $\Delta P_{\mu s}$ in other oscillation channels that are challenging to study, are large. ${ }^{3}$

In figure 3 , we show the spread of $A_{\nu \bar{\nu}},{ }^{4}$ at $L=1300 \mathrm{~km}$ for cases chosen to illustrate some of the important features that arise due to the presence of a fourth, sterile state. The left-hand panels were created with all CP-violating phases set to zero, so the asymmetries shown in these panels are from matter effects only. The right-hand panels were created allowing the sole $3+0 \mathrm{CP}$ phase $\delta_{\mathrm{CP}}$ to vary over its entire physical range in the case of $3+0$, and the three CP phases $\delta_{13}, \delta_{24}$ and $\delta_{34}$ to vary over their entire ranges in the case of $3+1$. Thus, these panels show the impact of intrinsic CP violation. In all panels, the red curve(s) are for the $3+0$ case, and the blue ones for the $3+1$ case. The top two panels assume a normal hierarchy, and the bottom two an inverted hierarchy. In creating all panels, the mass splittings and mixing angles of the $3+0$ sector were set to the best-fit values specified in section 4 , the splitting $\Delta m_{41}^{2}$ of the $3+1$ sector was set to $1 \mathrm{eV}^{2}$, and the $3+1$ mixing angles $\theta_{14}, \theta_{24}$ and $\theta_{34}$ were varied over their allowed ranges.

With one exception (see below), to create the curves in each panel of figure 3 for each of the two scenarios, $3+0$ and $3+1$, we varied the corresponding parameters until we found the parameter set that maximizes (minimizes) the energy-integrated asymmetry $A_{\nu \bar{\nu}}$ for that

\footnotetext{
${ }^{3}$ We note that any long baseline experiment involves earth-matter effects, which break CPT (in addition to $\mathrm{CP}$ ). Such breaking is extrinsic, and due to the asymmetry of the earth matter through which the neutrinos propagate. While this may appear to destroy the conclusions reached above, which depend on CPT invariance, this is not the case as long as an experiment seeks to measure intrinsic (i.e. driven by phases in the mixing matrix) $\mathrm{CP}$ violation and devises appropriate means to do so.

${ }^{4}$ Henceforth we drop the superscripts $\alpha$ and $\beta$ and take $A_{\nu \bar{\nu}}$ to denote the asymmetry for $\alpha=\mu$ and $\beta=e$.
} 
scenario. The energy-dependent asymmetry was then plotted vs. energy for this parameter set as a solid (dashed) curve. (Note that since it is the energy-integrated asymmetry that is being extremized, it is possible for the $3+0$ energy-dependent asymmetry to be more extreme than that for $3+1$ for a limited range of energy, despite the fact that the $3+0$ scenario is in a sense, a special case of $3+1$.) The one exception to our procedure is that, since the $3+0$ sector parameters other than $\delta_{\mathrm{CP}}$ were held fixed throughout, in creating the left-hand panels, no $3+0$ parameters were varied, so there is only a single curve, shown as solid, for $3+0$.

From the left-hand panels of figure 3, we see that when CP is conserved, the neutrinoantineutrino asymmetry vs. energy is quite similar in the $3+0$ and $3+1$ scenarios. In the $3+1$ scenario, this asymmetry is confined to a rather narrow band as the $3+1$ mixing angles are varied. Although it is not shown, we have found that it is confined to a similar narrow band in the $3+0$ scenario as the $3+0$ parameters $\theta_{23}$ and $\Delta m_{31}^{2}$ are varied within their experimental uncertainties. Clearly, if an experiment were to measure an asymmetry vs. energy that consistently lies outside the similar, narrow $3+0$ and $3+1$ bands that correspond to $\mathrm{CP}$ conservation, we would have evidence that $\mathrm{CP}$ is violated so long as nature has chosen either the $3+0$ or $3+1$ scenario. However, a measured asymmetry between $\nu_{\mu} \rightarrow \nu_{e}$ and $\bar{\nu}_{\mu} \rightarrow \bar{\nu}_{e}$ that lies within these similar narrow bands would not unambiguously signal that there is no $\mathrm{CP}$ violation in neutrino oscillation. As explained above, when there are more than three flavors, as in the $3+1$ scenario, it is possible for there to be little or no $\mathrm{CP}$ violation in one oscillation channel, and yet a large $\mathrm{CP}$ violation in some other channel. In addition, for either the $3+0$ or $3+1$ case, it might happen that for some non-zero values of the $\mathrm{CP}$-violating phases and mixing angles slightly different from those corresponding to the CP-conserving bands, the asymmetry still lies within those bands within uncertainties.

The right-hand panels in figure 3 show that when intrinsic CP is violated, $A_{\nu \bar{\nu}}$ can be anywhere in a large range. Moreover, for $3+1$, this range is much larger than for $3+0$, and includes almost all of the $3+0$ range. Thus, we see that sterile neutrinos with $O(1)$ $\mathrm{eV}^{2}$ masses can very substantially impact $\mathrm{CP}$-violation measurements at long baselines. While a measured asymmetry outside the band allowed for $3+0$ would be evidence for new physics beyond $3+0$, one inside that band would leave uncertain the precise origin of the observed $\mathrm{CP}$ violation.

To understand why the effect of a fourth, sterile neutrino on $\mathrm{CP}$ violation at long baselines can be so large, one notes that $\mathrm{CP}$-violating phases affect physics through interferences between amplitudes. As pointed out in [26], around the first maximum of the atmospheric-wavelength oscillation, where the long-baseline experiments work, or will work, the (new, short wavelength oscillation) - (atmospheric-wavelength oscillation) interference, and the (atmospheric-wavelength oscillation) - (solar-wavelength oscillation) interference, can easily be of comparable size. Then, if the $\mathrm{CP}$ phases are in the right range, $3+1$ can be quite different from $3+0$.

In the next section, we see how the probability-level results of this section and the previous one translate into observable consequences for DUNE by calculating event rates. 


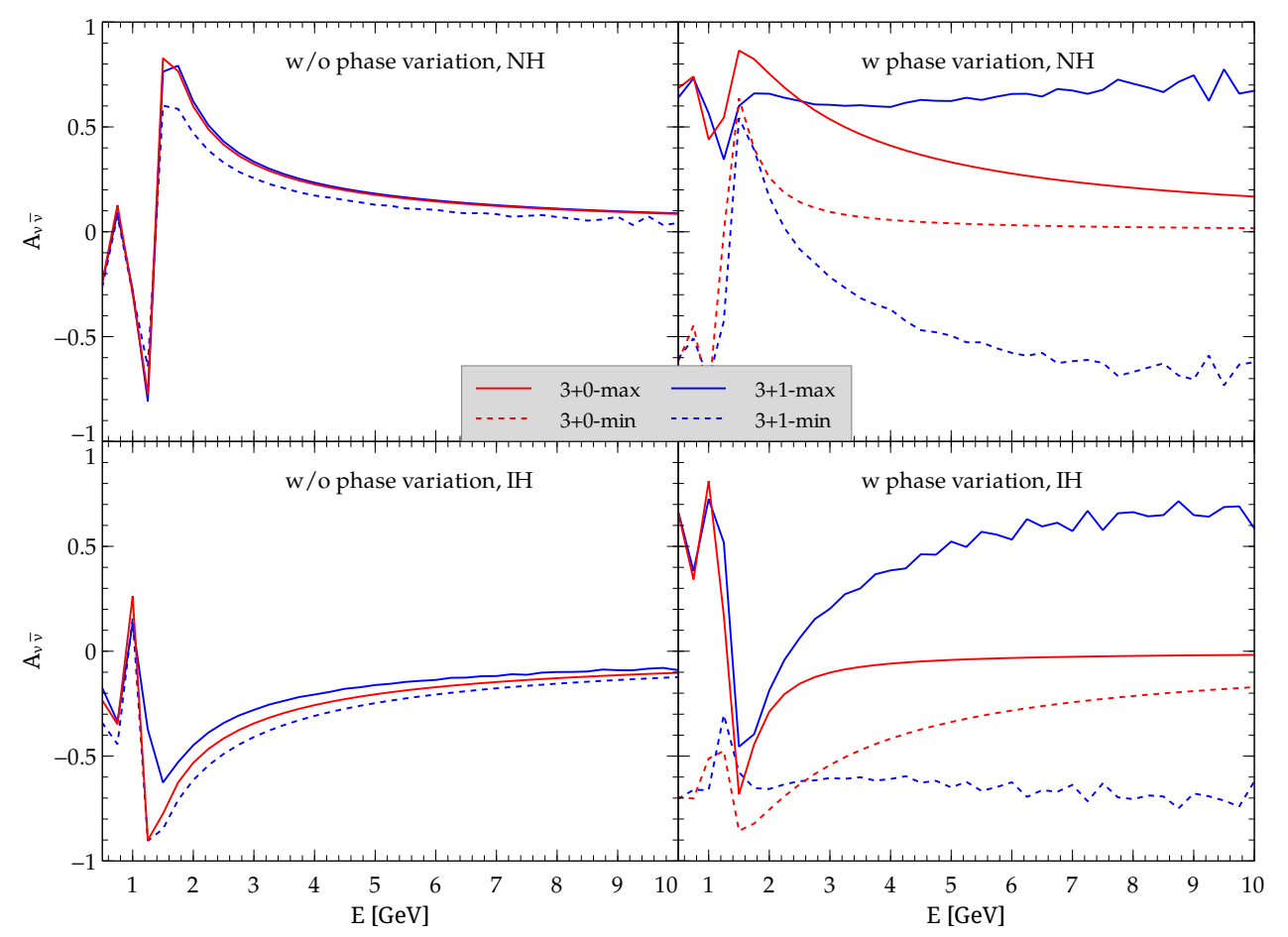

Figure 3. The neutrino-antineutrino asymmetry $A_{\nu \bar{\nu}}$ vs. energy E. See text for explanation and discussion.

\section{Event rates at DUNE in the $3+1$ and $3+0$ scenarios}

Having discussed some of the salient features of the probability $P_{\mu e}^{4 \nu}$ both in vacuum and matter at baselines characteristic of DUNE, we now perform event rate calculations by realistically simulating the experiment.

We recall that DUNE (with specifications very similar to LBNE, $[18,19]$ ) is a proposed future super-beam experiment, to be located in the United States, with a main aim of establishing or refuting the existence of $\mathrm{CPV}$ in the leptonic sector. In addition to this primary goal, this facility will also be able to resolve other important issues like the mass hierarchy and the octant of $\theta_{23}$. The $\nu_{\mu}\left(\bar{\nu}_{\mu}\right)$ super-beam will originate at the Fermilab. The primary beam simulation assumes a $1.2 \mathrm{MW}-120 \mathrm{GeV}$ proton beam which will deliver $10^{21}$ protons-on-target (POT) per year. A 35-40 kt Liquid Argon (LAr) far-detector will be housed at the Sanford Underground Research Facility in the Homestake mine in South Dakota, $1300 \mathrm{~km}$ away. The experiment plans to have a total of 10 years of running, divided equally between neutrinos and anti-neutrinos, corresponding to a total exposure of $35 \times 10^{22}$ kt-POT-yr. The other experimental details such as signal and background definitions as well as the detector efficiencies taken in this work are the same as those in [20], except with the difference that we have not considered tau events in the backgrounds. The detector efficiencies for both $P_{\mu e}$ and $P_{\bar{\mu} \bar{e}}$ events are close to $80 \%$ with somewhat less efficiency for $P_{\bar{\mu} \bar{e}}$.

In order to facilitate the drawing of physics conclusions, we assume certain values and ranges for neutrino oscillation parameters in the standard 3-flavour paradigm, which are 
motivated by their current measured ranges and best fit values. Specifically,

- $\theta_{12}$ and $\theta_{13}$ have been fixed at $33.48^{\circ}$ and $8.5^{\circ}$ respectively [35].

- We assume that the $2-3$ mixing is near-maximal i.e. $\theta_{23}=45^{\circ} .^{5}$

- We fix $\Delta m_{21}^{2}$ at $7.5 \times 10^{-5} \mathrm{eV}^{2}$ and $\Delta m_{31}^{2}$ at $2.457 \times 10^{-3} \mathrm{eV}^{2}\left(-2.374 \times 10^{-3} \mathrm{eV}^{2}\right)$ for $\mathrm{NH}(\mathrm{IH})$. These particular choices have been taken from the analysis of global data [35].

We note that as stated above, standard global analyses ([35-37]) assume the $3+0$ scenario, but, as has been demonstrated in e.g. [38], their conclusions remain very robust in the presence of sterile neutrinos. Our assumed ranges for the sterile sector mixing angles corresponding to the $3+1$ scenario are as stated in section 2 . In addition, we assume $\Delta m_{41}^{2}$ to be $1 \mathrm{eV}^{2}$, and vary $\delta_{13}, \delta_{24}$ and $\delta_{34}$ for $3+1$ and $\delta_{\mathrm{CP}}$ for $3+0$ in the entire possible range of $\left[-180^{\circ}, 180^{\circ}\right]$. Finally, we use the fluxes provided in [39].

Figure 4 shows the spread of binned events as a function of the reconstructed neutrino energy for the $3+0$ case and the $3+1$ case. For the $3+0$ case, we varied only $\delta_{\mathrm{CP}}$ in the range $\left[-180^{\circ}, 180^{\circ}\right]$ to obtain the events band shown in red. For $3+1$, we chose three sets of $\theta_{14}, \theta_{24}$ values $-\left(20^{\circ}, 10^{\circ}\right),\left(15^{\circ}, 10^{\circ}\right)$ and $\left(5^{\circ}, 5^{\circ}\right)$. For all these three cases, we varied $\theta_{34}$ in the range $\left[0,30^{\circ}\right]$ and the phases $\delta_{13}, \delta_{24}$ and $\delta_{34}$ in the range $\left[-180^{\circ}, 180^{\circ}\right]$ each. The resulting event-bands are shown in blue, green and magenta, respectively. The left (right) panels show the neutrino (anti-neutrino) rates, while the top (bottom) panels are for the $\mathrm{NH}(\mathrm{IH})$ scenario.

It can be seen that for all three sets of $\theta_{14}, \theta_{24}$, the $3+1$ band can potentially encompass the $3+0$ band, leading to substantial degeneracy. When the number of events falls in the overlapping region between these two bands (which is the red region in figure 4), there is considerable ambiguity as to whether the events are produced by a certain value of $\delta_{\mathrm{CP}}$ in the $3+0$ sector or by some combination of $\theta_{34}, \delta_{13}, \delta_{24}$ and $\delta_{34}$ in the $3+1$ sector.

Figure 4 also shows that the $3+1$ band gets wider as the values of $\theta_{14}, \theta_{24}$ and hence the effective mixing angle $\sin 2 \theta_{\mu e}^{4 \nu}$ increase. Indeed, for sufficiently large $3+1$ mixing angles, the $3+1$ band is substantially larger than its $3+0$ counterpart. An observed surfeit or a dearth of events compared to those expected in the $3+0$ case, especially near the event maxima (around the region $2-4 \mathrm{GeV}$ ), could be a pointer to the presence of sterile states.

\section{Implications}

Prior to summarizing our conclusions, it is useful to discuss certain implications which arise from our results. We have noted above that in the presence of even a single sterile neutrino, conclusions such as, a) Whether CP is conserved or violated, and b) if the latter, whether the violation is ascribable to the active neutrinos or the additional sterile neutrino,

\footnotetext{
${ }^{5}$ We note that the currently-allowed $3 \sigma$ range on $\theta_{23}$ is $\left[38.3^{\circ}, 53.3^{\circ}\right]$ with the best fit at $42.3^{\circ}(49.5)^{\circ}$ for $\mathrm{NH}(\mathrm{IH})$ [35]. The $\theta_{23}$ best fit values from the global analyses [36, 37] are different from [35]. However, in this work, we make the simplifying assumption of maximal mixing, relegating a more rigorous statistical analysis to a follow-up work.
} 

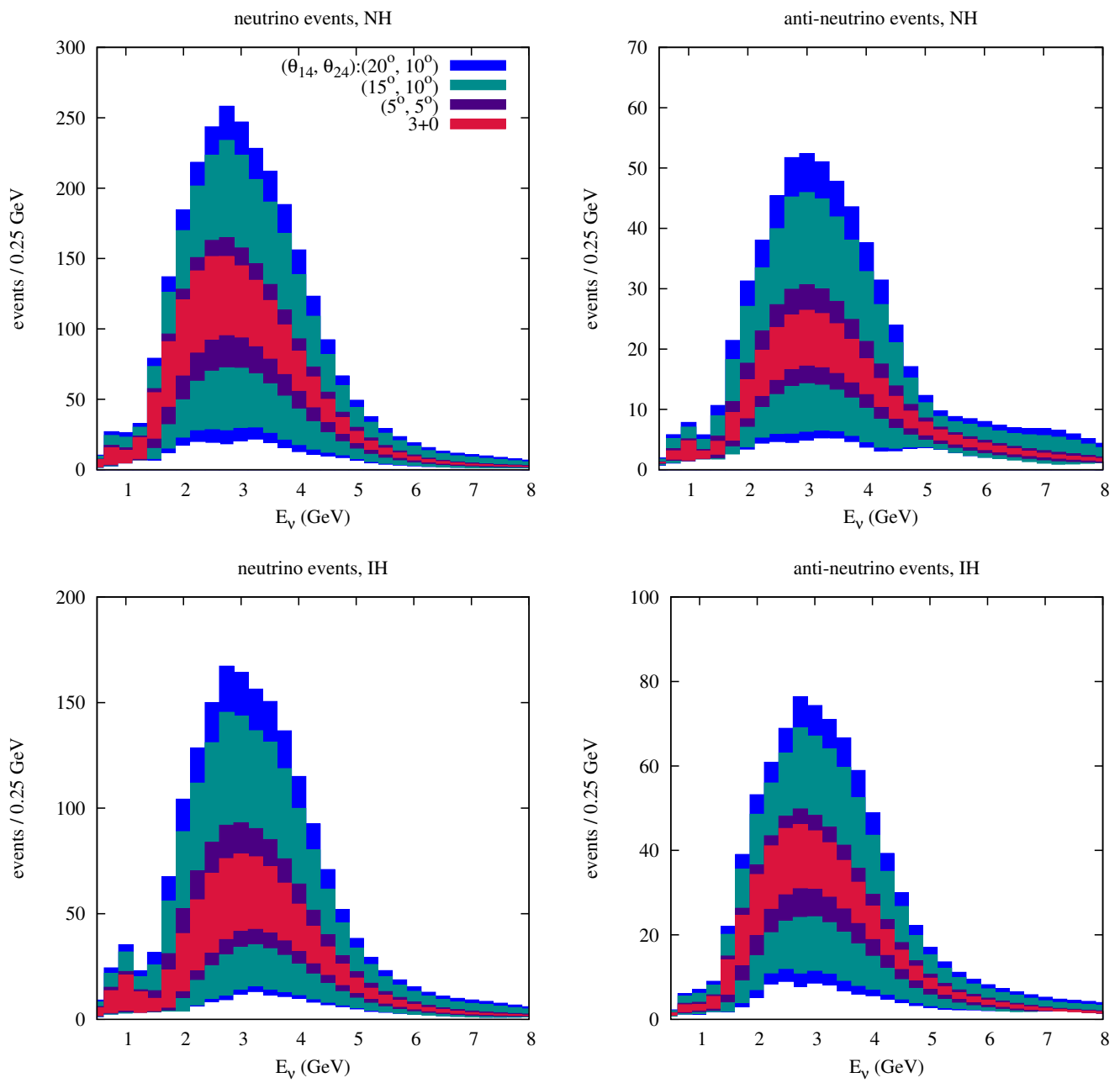

Figure 4. Neutrino and anti-neutrino event rates in DUNE plotted as a function of the reconstructed neutrino energy. The vertical spread for a given color for an energy bin shows the maximum and the minimum events rates possible.

or a combination of the two, are all rendered significantly ambiguous. An important consequence is thus the need for an improved synergistic linkage between the global LBL and SBL efforts [40], since it appears that results obtained in the former cannot be correctly interpreted without definitive conclusions drawn from the latter.

Our work also has ramifications for near detector (ND) design and physics requirements in DUNE and other LBL experiments. Even if there is no sterile sector and the standard three-family scenario is nature's choice, in order to fully exploit the CPV capabilities of a far detector (FD), the ND must establish the expected number of events at the FD in the absence of oscillations with very high precision, in order to ensure systematic errors stay well below statistical ones. The rates expected at the FD depend on fluxes and cross-sections measured, along with their energy dependence, to significantly high accuracy at the ND for all four species of neutrinos, $\nu_{e}, \bar{\nu}_{e}, \nu_{\mu}, \bar{\nu}_{\mu}$. In the $3+0$ scenario, these measurements, while very demanding, are assumed to be made under conditions where there are no oscillations 
between the source and the ND. This task is rendered significantly more complex, however, in the presence of a sterile sector capable of altering the fluxes between the source and the ND over the planned distance of $\sim 500 \mathrm{~m}$ in DUNE. Historically, uncertainties in source fluxes and cross-sections have always been a major limiting factor for neutrino experiments seeking precision in oscillation studies. The high intensity of the DUNE beam and the presence of a highly capable and precise ND, designed to overcome these limitations, are thus rendered even more crucial than assumed earlier in order to deconvolute the added complexities arising due to a sterile sector, if it were to be present in nature. Moreover, we note that current data allow the mass-squared splitting in the sterile sector to be sufficiently large such that oscillations due to it can average out even at the ND distance of $\sim 500 \mathrm{~m}$. Such an eventuality further underscores the need for a strong connection between the SBL and LBL experimental programs, since these oscillations, even though not directly observed at the ND, would nonetheless affect its crucial service task of accurate flux determination.

\section{Summary and conclusions}

To summarize, we have studied the effects of the additional mixing angles and CP phases in the case of a $3+1$ sterile sector on the determination and measurement of CPV at long baselines for the DUNE experiment. From a probability analysis, we show that the effects of the additional CP phases can be large at its chosen baseline of $1300 \mathrm{~km}$. These effects, which arise from large interference terms (between the $3+0$ and $3+1$ sectors) in the appearance probability, are accentuated by the presence of matter, which additionally brings in contributions from sterile-sector mixings and phases which are dormant at short baselines. From event rate calculations, we show that the presence of a sterile sector manifests itself in measurably altered rates in energy bins across the spectrum, without significant distortion in the shape. This alteration in event rates increases, as expected, for larger values of the mixing angles connecting the active and sterile sectors.

Importantly, the presence of a sterile sector obfuscates conclusive determinations of $\mathrm{CP}$ violation or conservation at the far detector, and makes uncertain the ability to ascribe any perceived CPV to a unique phase in the $3+0$ sector. Thus, the linkage between the presently planned long and short baseline programs must be explored and strengthened. Until the presence of an $\sim \mathrm{eV}^{2}$ sector is conclusively ruled out, our work emphasizes the need for a complementary SBL sterile-search program and for a highly capable and versatile near detector for DUNE, enabling it to reduce systematics to low levels so that it may achieve its stated primary goals for CPV detection.

\section{Acknowledgments}

We thank Mary Bishai for help with DUNE fluxes and for patiently answering questions related to DUNE. RG and BK thank William Louis for very useful discussions. RG acknowledges useful discussions with Sandhya Choubey, Amol Dighe and S. Uma Sankar. MM thanks Animesh Chatterjee for very helpful discussions. RG and BK are grateful to the Kavli Institute for Theoretical Physics for support and hospitality. RG acknowledges 
support from the Fermilab Neutrino Division and the DUNE Project at Fermilab while this work was in progress in the form of an Intensity Frontier Fellowship in 2014 and a Guest Scientist position in 2015. RG, MM and SP also acknowledge support from the XII Plan Neutrino Project of the Indian DAE. BK thanks the Mainz Institute for Theoretical Physics for its hospitality and support while this paper was completed. This research was supported in part by the US National Science Foundation under Grant No. NSF PHY11-25915. Fermilab is operated by Fermi Research Alliance, LLC under Contract No. DE-AC02-07CH11359 with the US Department of Energy.

Open Access. This article is distributed under the terms of the Creative Commons Attribution License (CC-BY 4.0), which permits any use, distribution and reproduction in any medium, provided the original author(s) and source are credited.

\section{References}

[1] M. Thomson, talk at the Workshop on the Intermediate Neutrino Program (WINP 2015), Brookhaven National Laboratory, Upton New York U.S.A., February 4-6 2015 [INSPIRE] and online at https://indico.bnl.gov/conferenceDisplay.py?confId=918.

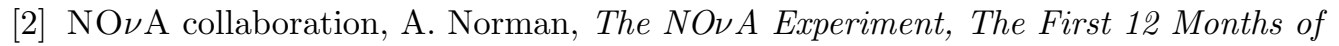
Commissioning, Operations and Physics Data, AIP Conf. Proc. 1666 (2015) 110001 [INSPIRE].

[3] C. Farnese, Some recent results from ICARUS, AIP Conf. Proc. 1666 (2015) 110002 [INSPIRE].

[4] OPERA collaboration, S. Dusini, Observation of $\nu_{\mu} \rightarrow \nu_{\tau}$ oscillations by the OPERA experiment, AIP Conf. Proc. 1666 (2015) 110003 [INSPIRE].

[5] MINOS+ and MINOS collaborations, A.B. Sousa, First MINOS+ Data and New Results from MINOS, AIP Conf. Proc. 1666 (2015) 110004 [arXiv: 1502.07715] [INSPIRE].

[6] A.K. Ichikawa, High intensity neutrino beams, AIP Conf. Proc. 1666 (2015) 130001 [INSPIRE].

[7] F.J.P. Soler, Neutrino Factories, AIP Conf. Proc. 1666 (2015) 130002 [inSPIRE].

[8] Y. Hayato, Future long-baseline neutrino oscillations: View from Asia, AIP Conf. Proc. 1666 (2015) 130003 [inSPIRE].

[9] LBNE collaboration, R.J. Wilson, Future Long-Baseline Neutrino Oscillations: View from North America, AIP Conf. Proc. 1666 (2015) 130004 [INSPIRE].

[10] T. Patzak, Future long-baseline neutrino oscillations: View from Europe, AIP Conf. Proc. 1666 (2015) 130005 [INSPIRE].

[11] ICFA Neutrino PAnel collaboration, K. Long, ICFA neutrino panel report, AIP Conf. Proc. 1666 (2015) 130006 [INSPIRE].

[12] Intensity Frontier Neutrino Working Group, A. de Gouvêa et al., Working Group Report: Neutrinos, arXiv:1310.4340 [INSPIRE].

[13] LSND collaboration, A.A. Aguilar-Arevalo et al., Evidence for neutrino oscillations from the observation of $\bar{\nu}_{e}$ appearance in a $\bar{\nu}_{\mu}$ beam, Phys. Rev. D 64 (2001) 112007 [hep-ex/0104049] [INSPIRE]. 
[14] MiniBoonE collaboration, A.A. Aguilar-Arevalo et al., Unexplained Excess of Electron-Like Events From a 1-GeV Neutrino Beam, Phys. Rev. Lett. 102 (2009) 101802 [arXiv:0812.2243] [INSPIRE].

[15] G. Mention et al., The Reactor Antineutrino Anomaly, Phys. Rev. D 83 (2011) 073006 [arXiv:1101.2755] [INSPIRE].

[16] T.A. Mueller et al., Improved Predictions of Reactor Antineutrino Spectra, Phys. Rev. C 83 (2011) 054615 [arXiv:1101.2663] [INSPIRE].

[17] MiniBoonE collaboration, A.A. Aguilar-Arevalo et al., Improved Search for $\bar{\nu}_{\mu} \rightarrow \bar{\nu}_{e}$ Oscillations in the MiniBooNE Experiment, Phys. Rev. Lett. 110 (2013) 161801 [arXiv: 1207.4809] [INSPIRE].

[18] J. Hewett et al., Fundamental Physics at the Intensity Frontier, arXiv:1205.2671 [doi:10.2172/1042577].

[19] LBNE collaboration, C. Adams et al., The Long-Baseline Neutrino Experiment: Exploring Fundamental Symmetries of the Universe, arXiv:1307.7335 [INSPIRE].

[20] LBNE collaboration, M. Bass et al., Baseline optimization for the measurement of CP-violation, mass hierarchy and $\theta_{23}$ octant in a long-baseline neutrino oscillation experiment, Phys. Rev. D 91 (2015) 052015 [arXiv:1311.0212] [INSPIRE].

[21] A. Donini, M. Lusignoli and D. Meloni, Telling three neutrinos from four neutrinos at the neutrino factory, Nucl. Phys. B 624 (2002) 405 [hep-ph/0107231] [INSPIRE].

[22] A. Dighe and S. Ray, Signatures of heavy sterile neutrinos at long baseline experiments, Phys. Rev. D 76 (2007) 113001 [arXiv:0709.0383] [inSPIRE].

[23] A. Donini, K.-i. Fuki, J. Lopez-Pavon, D. Meloni and O. Yasuda, The Discovery channel at the Neutrino Factory: $\nu_{\mu} \rightarrow \nu_{\tau}$ pointing to sterile neutrinos, JHEP 08 (2009) 041 [arXiv: 0812.3703] [INSPIRE].

[24] O. Yasuda, Sensitivity to sterile neutrino mixings and the discovery channel at a neutrino factory, arXiv: 1004.2388 [INSPIRE].

[25] D. Meloni, J. Tang and W. Winter, Sterile neutrinos beyond LSND at the Neutrino Factory, Phys. Rev. D 82 (2010) 093008 [arXiv:1007.2419] [INSPIRE].

[26] N. Klop and A. Palazzo, Imprints of CP-violation induced by sterile neutrinos in T2K data, Phys. Rev. D 91 (2015) 073017 [arXiv: 1412.7524] [INSPIRE].

[27] T2K collaboration, K. Abe et al., Observation of Electron Neutrino Appearance in a Muon Neutrino Beam, Phys. Rev. Lett. 112 (2014) 061802 [arXiv:1311.4750] [INSPIRE].

[28] B. Bhattacharya, A.M. Thalapillil and C.E.M. Wagner, Implications of sterile neutrinos for medium/long-baseline neutrino experiments and the determination of $\theta_{13}$, Phys. Rev. D 85 (2012) 073004 [arXiv:1111.4225] [INSPIRE].

[29] MINOS collaboration, P. Adamson et al., Combined analysis of $\nu_{\mu}$ disappearance and $\nu_{\mu} \rightarrow \nu_{e}$ appearance in MINOS using accelerator and atmospheric neutrinos, Phys. Rev. Lett. 112 (2014) 191801 [arXiv:1403.0867] [INSPIRE].

[30] D. Hollander and I. Mocioiu, Minimal $3+2$ sterile neutrino model at LBNE, Phys. Rev. D 91 (2015) 013002 [arXiv: 1408.1749] [INSPIRE].

[31] J.M. Berryman, A. de Gouvêa, K.J. Kelly and A. Kobach, Sterile neutrino at the Deep Underground Neutrino Experiment, Phys. Rev. D 92 (2015) 073012 [arXiv:1507.03986] [INSPIRE]. 
[32] J. Kopp, P.A.N. Machado, M. Maltoni and T. Schwetz, Sterile Neutrino Oscillations: The Global Picture, JHEP 05 (2013) 050 [arXiv: 1303.3011] [INSPIRE].

[33] P. Huber, M. Lindner and W. Winter, Simulation of long-baseline neutrino oscillation experiments with GLoBES (General Long Baseline Experiment Simulator), Comput. Phys. Commun. 167 (2005) 195 [hep-ph/0407333] [INSPIRE].

[34] P. Huber, J. Kopp, M. Lindner, M. Rolinec and W. Winter, New features in the simulation of neutrino oscillation experiments with GLoBES 3.0: General Long Baseline Experiment Simulator, Comput. Phys. Commun. 177 (2007) 432 [hep-ph/0701187] [INSPIRE].

[35] M.C. Gonzalez-Garcia, M. Maltoni and T. Schwetz, Updated fit to three neutrino mixing: status of leptonic CP-violation, JHEP 11 (2014) 052 [arXiv: 1409.5439] [INSPIRE].

[36] F. Capozzi, G.L. Fogli, E. Lisi, A. Marrone, D. Montanino and A. Palazzo, Status of three-neutrino oscillation parameters, circa 2013, Phys. Rev. D 89 (2014) 093018 [arXiv: 1312.2878] [INSPIRE].

[37] D.V. Forero, M. Tortola and J.W.F. Valle, Neutrino oscillations refitted, Phys. Rev. D 90 (2014) 093006 [arXiv: 1405.7540] [INSPIRE].

[38] A. Esmaili, E. Kemp, O.L.G. Peres and Z. Tabrizi, Probing light sterile neutrinos in medium baseline reactor experiments, Phys. Rev. D 88 (2013) 073012 [arXiv: 1308.6218] [INSPIRE].

[39] M. Bishai, private communication (2014).

[40] J. Zennamo, The Short-Baseline Neutrino Program, in $48^{\text {th }}$ Annual Fermilab User's Meeting, Fermilab Ramsey Auditorium, Batavia Illinois U.S.A., 9-11 June 2015, https://indico.fnal.gov/getFile.py/access?contribId $=54 \&$ sessionId $=23 \&$ resId $=0 \&$ materialId $=$ slides\&confId $=8982$. 